\title{
Laparoscopic surgery for pancreatic insulinomas: an update
}

\author{
Chrysanthi Aggeli, ${ }^{1}$ Alexander M. Nixon, ${ }^{1}$ Ioannis Karoumpalis, ${ }^{2}$ \\ Gregory Kaltsas, ${ }^{3}$ George N. Zografos ${ }^{1}$
}

${ }^{1}$ Third Department of Surgery, 'Department of Gastroenterology, Athens General Hospital "Georgios Gennimatas", Athens, ${ }^{3}$ Department of Pathophysiology, Laiko Hospital, Athens Greece

\begin{abstract}
Insulinomas are the most common functioning neuroendocrine tumors of the pancreas, occurring in almost 1-4 per 1 million persons each year. In contrast to other pancreatic neuroendocrine tumors, they are usually benign and solitary at the time of diagnosis. Due to their benign nature, surgical excision is the treatment of choice, with excellent long-term results. The introduction of minimally invasive techniques in the surgical treatment of insulinoma has been gaining popularity due to shorter length of hospital stay and better cosmetic results, with serious complications being comparable to those of open surgery. Preoperative localization is of paramount importance in the determination of the appropriate surgical approach. Many invasive and non-invasive methods exist for localization of an insulinoma. A combination of these modalities is usually adequate to preoperatively localize the vast majority of tumors. Laparoscopic ultrasound is mandatory to localize these tumors intraoperatively. Despite extensive experience in highly specialized centers producing encouraging results, no randomized trials have been realized to conclusively validate these case series, this partly due to the rarity of insulinoma in the population. In this article we present the current state of laparoscopic management of insulinoma delineating still unanswered issues and we underscore some of the technical details of the most common laparoscopic procedures employed.
\end{abstract}

Key words: EUS, Enucleation, Insulinoma, Pancreatectomy

\section{INTRODUCTION}

Pancreatic neuroendocrine tumors (PanNETs) are a rare group of neoplasms that originate from the endocrine portion of the pancreas. These tumors are

Address for correspondence:

Chrysanthi Aggeli, MD, PhD, Consultant in Surgery, National

Healthcare Service (ESY), Athens General Hospital "Georgios

Gennimatas", 154 Mesogeion Avenue, Athens, 11527, Greece,

Tel.: +30-210-7768634, Fax: +30-210-7706915,

E-mail: chraggeli@gmail.com

Received: 12-05-2016, Accepted: 26-05-2016 further classified depending on whether they secrete compounds, resulting in symptoms, into functioning and non-functioning PanNETs. ${ }^{1}$ Insulinoma was the first PanNET to be identified nearly 90 years ago and it represents the majority of functioning PanNETs. The name denotes the tumor's biological behavior and propensity to secrete considerable amounts of insulin, leading to clinical manifestations of hypoglycemia.

PanNETs as a whole constitute only about $1-2 \%$ of pancreatic tumors. ${ }^{2}$ Taking this into consideration, it is not surprising that attempts to estimate insulinoma 
incidence in the general population have been beset with difficulties. Studies based on autopsy reports have indicated that clinically indolent insulinomas are exceedingly rare. ${ }^{3}$ Most epidemiological information regarding the incidence of insulinomas in the general population is derived from Olmsted County, Minnesota, USA, and a retrospective study spanning six decades where incidence of insulinoma was estimated at 4 cases per million per year. ${ }^{4}$ Of note is the upward trend in incidence in the last two decades of the study. This upward trend has been observed as regards several varieties of neuroendocrine tumors of the digestive tract, most likely indicating a refinement in diagnostic techniques.

Based on available patient series, there does not seem to be a predilection concerning sex, with insulinomas afflicting both genders equally. When sporadic (approximately 90\%), insulinomas tend to be solitary tumors that can be treated effectively with limited resections. ${ }^{4}$ By contrast, in multiple endocrine neoplasia (MEN) type 1 syndrome (4-10\%) insulinomas tend to be multiple, have malignant potential (up to $25 \%$ ) and usually require more complicated and extensive resections of pancreatic parenchyma. ${ }^{4-6}$ Conversely, approximately $10 \%$ of patients with MEN1 syndrome develop insulinomas.

Although sporadic insulinoma may occur throughout life, the mean age at presentation is 47 years (range 8-82). ${ }^{7}$ In MEN1 the mean age at presentation is lower, at 25 years or less. Anatomical distribution may vary throughout the head, body and tail of the pancreas, but they are predominantly located in the body and the tail. ${ }^{4,6,8}$ The majority $(90 \%)$ have a diameter smaller than $2 \mathrm{~cm}$, whereas malignant tumors are usually over $3 \mathrm{~cm} .{ }^{9}$ Due to the fact that symptoms of hypoglycemia dominate the clinical picture early in the course of the disease the majority are small at the time of presentation and amenable to surgical resection.

Clinical suspicion of insulinoma is warranted in cases of hypoglycemia related symptoms, with approximately $75 \%$ of patients reporting symptoms in the fasting state. ${ }^{10}$ However, postprandial hypoglycemia can occur in conjunction with fasting hypoglycemia and in a minority of patients may be the only symptom. ${ }^{11}$ Symptoms are categorized into the neuroglycopenic type, which result from dysfunction of the central nervous system due to a lack of glucose, its primary energy substrate, and the sympathoadrenal type which occur as part of the stress response and the subsequent release of catecholamines. During episodes of hypoglycemia patients may experience blurred vision and exhibit erratic behavior. Due to stimulation of the autonomic nervous system, diaphoresis, palpitations and excitability are all common presentations. ${ }^{11}$ In extreme cases excessive hypoglycemia may result in seizures and a comatose state.

Historically, Whipple's triad has summarized the clinical picture and diagnosis of insulinoma (induction of the symptoms after fasting or exercise, a plasma glucose $<50 \mathrm{mg} / \mathrm{dl}$ when symptomatic, relief of symptoms after administration of oral or intravenous glucose) and is still employed today to diagnose hypoglycemic disorders in non-diabetic patients. ${ }^{12}$ Diagnosis of an insulinoma is dependent on documenting inappropriately elevated levels of insulin in the setting of low circulating levels of glucose. Specific criteria have been developed dependent on blood based tests to confirm the diagnosis. ${ }^{13}$ Once a state of incongruent hyperinsulinemia has been diagnosed, localization studies are necessary to visualize the tumor.

Therapeutic strategies for both benign and malignant insulinomas typically involve surgical management and/or percutaneous ablation of lesions, and in the case of metastatic disease chemotherapy. ${ }^{13,14}$ The first surgical procedure for the treatment of insulinoma was described in $1929 .{ }^{12}$ Depending on lesion localization and extent of disease, surgical options include enucleation and/or excision of pancreatic parenchyma. Before the advent of minimally invasive surgical techniques and advanced imaging modalities, even simple enucleations in readily accessible areas of the pancreas entailed exposure of the pancreas through large abdominal incisions. Not uncommonly, difficulty or uncertainty in intraoperative identification of lesions leads to conversion of a relatively simple procedure such as enucleation to extensive procedures (e.g. Whipple's procedure) with high morbidity and mortality. Laparoscopic surgery and preoperative and intraoperative imaging modalities have completely transformed the treatment of insulinomas. Preservation of the pancreatic parenchyma is currently considered mandatory, as insulinomas have an excellent potential 
for complete cure after surgical excision and because most are small, solitary and benign. ${ }^{4} \mathrm{~A}$ combination of preoperative imaging tests (invasive and non-invasive) and intraoperative ultrasound have made laparoscopic procedures for insulinoma technically feasible and safe in centers with a high level of expertise. Here we summarize and comment on current advances in the laparoscopic management of insulinomas.

\section{LOCALIZATION OF THE TUMOR}

After detection of inappropriately high insulin levels imaging techniques are used to verify and localize the presence of a single or several insulinomas. Preoperative localization of the insulinoma is of utmost importance in the management of these neoplasms, since their location in the pancreas, their number and their relationship to important anatomical structures are the most important determinants of the surgical procedure selected. Traditionally, blind distal pancreatectomy used to be the standard surgical procedure when a tumor could not be visualized and/or palpated intraoperatively. However, advanced localization techniques have rendered blind resections for small and benign insulinomas obsolete. ${ }^{15}$ As previously mentioned, insulinomas are usually benign and most patients remain disease-free following successful excision, even after prolonged follow-up. Therefore, extensive pancreatic resections are not currently indicated in these scenarios due to the associated perioperative and postoperative morbidity and mortality of such procedures.

Several invasive and non-invasive imaging modalities are currently available and to a large degree choice of imaging test is dependent on local experience, availability and patient preference. Imaging studies provide crucial information regarding the location of the tumor, the extent of local invasion and the presence of metastatic lesions. The imaging studies include invasive and non-invasive tests for the preoperative localization of the insulinoma and laparoscopic ultrasound for intraoperative localization.

Non-invasive tests include spiral computed tomography (CT), magnetic resonance imaging (MRI), transabdominal ultrasound (US), pentetreotide scintigraphy and positron emission tomography (PET) with fluorine-18-L-dihydroxyphenylalanine. In certain cases, a transabdominal US is the first test employed due to its universal availability, low cost compared to other techniques, lack of radiation exposure, patient compliance and ability to evaluate possible abdominal metastasis. Major drawbacks include its dependence on operator expertise, difficulties in evaluating intra-abdominal structures in the obese patient and declining sensitivity with small diameter lesions $(<2$ $\mathrm{cm}$ ) and with tumors located in the tail of the pancreas. ${ }^{16,17}$ However, in certain high-volume centers detection by contrast-enhanced transabdominal US has been reported to be comparable to detection by conventional CT. ${ }^{18}$

$\mathrm{CT}$ is the most commonly used non-invasive preoperative localization test. Contrast enhancement is routinely used and insulinomas are usually visualized as rounded, well-defined lesions. Previous large-scale studies evaluating CT sensitivity in insulinoma detection have yielded modest results (approximately 70\%). ${ }^{19}$ However, developments in CT technology have drastically changed its usefulness as a first-line option in preoperative detection and previous modest results are probably not indicative of the current state of the technology. Dynamic CT has supplanted conventional CT scanning as the modality of choice due its ability to better detect small lesions thus increasing sensitivity to over $80 \% .{ }^{20,21}$ Large-scale single institution studies have documented considerable improvement of sensitivity with the adoption of newer CT techniques. ${ }^{21}$ Added benefits of CT compared to transabdominal US include better visualization of the abdominal cavity in order to exclude metastasis or concurrent abdominal pathology and less subjective interpretation of imaging findings. Surgeons well versed in abdominal CT scans can also better visually comprehend tumor localization, thus leading to better preoperative planning. Obvious drawbacks include radiation exposure and in a small subset of susceptible patients severe and possibly life-threatening allergic reactions to the contrast medium.

MRI is an additional study that can be employed, with various rates of success reported in the literature. ${ }^{22}$ Its major drawback is patient compliance, therefore limiting its use as a first choice. However, published results suggest that MRI is of particular importance in small diameter insulinomas that are not detected by transabdominal US and CT. ${ }^{23,24}$ In T1-weighted 
images insulinomas are low in signal intensity and relatively high in T2-weighted images (Figure 1). ${ }^{25}$

Scintigraphy with pentetreotide is of limited use in sporadic insulinoma, since most insulinomas do not readily express a large number of somatostatin receptors. Sensitivity is approximately $40-50 \%$ for insulinoma, but it is of use in hereditary syndromes such as MEN1 where there is a high likelihood of other PanNETs. ${ }^{24,26}$

Invasive tests include endoscopic ultrasound (EUS), selective angiography and selective arterial calcium stimulation (SACS). Selective angiography was traditionally employed in previous decades as the gold standard for preoperative localization. ${ }^{27}$ Introduction of non-invasive techniques with similar or superior diagnostic accuracy have rendered this modality mostly obsolete due to its invasive nature and potential for severe morbidity.

The SACS test constitutes an evolution of traditional transhepatic portal venous sampling. During this procedure, calcium gluconate is injected into the gastroduodenal, proper hepatic, splenic and superior mesenteric artery and hepatic venous blood is collected for evaluation of insulin levels. ${ }^{28}$ Selective catheterization of the aforementioned vessels permits reliable localization of insulinoma. However, anatomical variations of these vessels, which are not uncommon, can

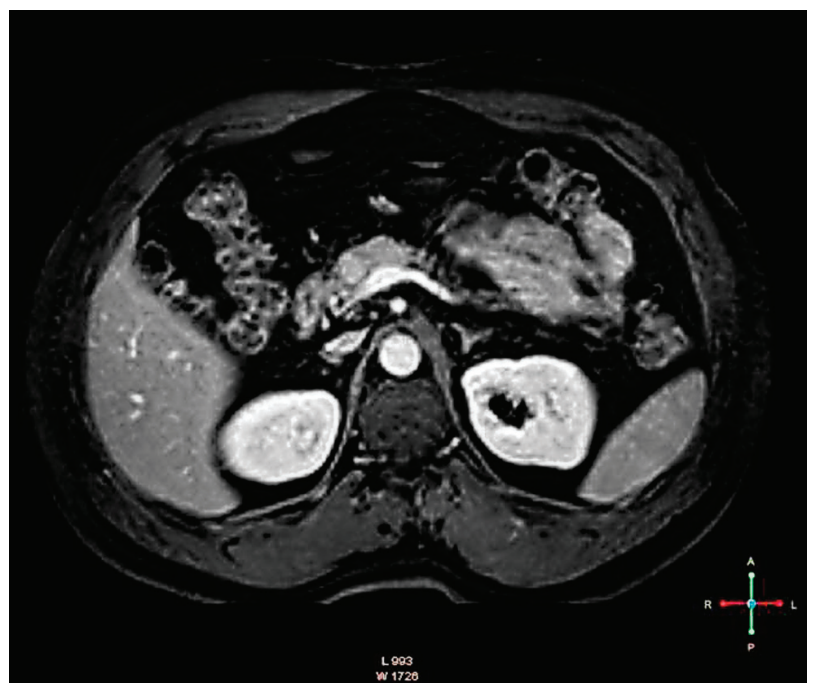

Figure 1. T1 weighted MRI visualizing a $2 \mathrm{~cm}$ insulinoma in the head of the pancreas. confound interpretation. ${ }^{29}$ SACS is of considerable importance in cases where other imaging modalities have failed to identify an insulinoma. In addition, it can be employed to distinguish between insulinoma and other very rare causes of hyperinsulinemic hypoglycemia such as nesidioblastosis. ${ }^{30,31}$ This test can distinguish whether insulin secretion is localized (as in the case of insulinoma) or spread throughout pancreatic tissue as in nesidioblastosis. Several reports have mentioned high levels of sensitivity reaching in some cases $100 \% .^{15,28,32}$ Obvious drawbacks include complications related to arterial catheterization, lack of availability and need for technical dexterity.

EUS, with reported sensitivity frequently over $80 \%,{ }^{13,26,33-35}$ is gradually gaining acceptance as an important adjuvant in preoperative evaluation of insulinoma and is considered the invasive test of choice if other non-invasive tests have failed to identify the tumor. EUS permits identification of lesions as small as $5 \mathrm{~mm}$ in diameter. ${ }^{33} \mathrm{As}$ is also the case in pancreatic adenocarcinoma, EUS yields important information regarding the lesion's proximity to major anatomic structures such as the major pancreatic duct and splenic vessels. ${ }^{36}$ EUS has been reported to be most accurate in tumors occurring in the head of the pancreas, while tumors at the tail are hardest to localize. ${ }^{37,38}$ Added benefits of EUS include the possibility for fine needle aspiration and cytological examination in circumstances where the diagnosis is in doubt and preoperative tattooing of the lesion/lesions to further assist in intraoperative identification (Figure 2) ${ }^{39-42}$ Furthermore, EUS guided alcohol ablation of insulinoma has been reported in high-risk surgical patients with encouraging results. ${ }^{43}$ Disadvantages of the technique include the need for adequate patient sedation and operator subjectiveness.

Currently there are no universally accepted guidelines as concerns preoperative imaging. Previous reports regarding non-invasive techniques have mostly involved older technologies that are steadily being replaced. In the case of CT and MRI, previous disappointing results are most certainly a reflection of that fact. Retrospective studies frequently included tests conducted by both multidetector and single-slice scanners, thereby probably underestimating the diagnostic utility of CT. ${ }^{24}$ At our institution, in sporadic insulinoma where lesions tend to be solitary and 


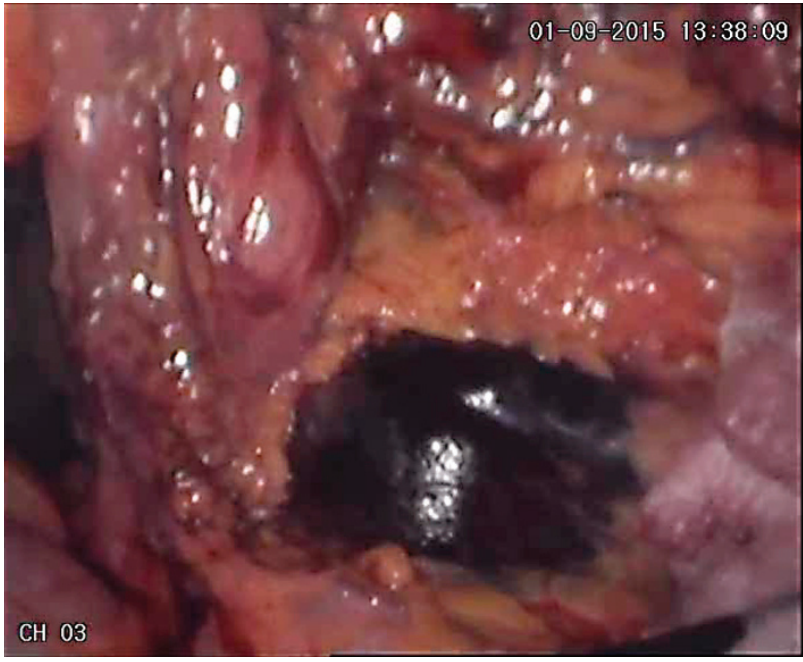

Figure 2. Laparoscopic view of an insulinoma on the anterior surface of the pancreas after tattooing with methylene blue dye by EUS.

benign we routinely utilize dynamic abdominal CT scan as the first imaging modality due to high patient compliance and its potential to recognize metastatic disease. We prefer to bypass transabdominal US based on the fact that transabdominal US images are not as readily intelligible as $\mathrm{CT}$ images for the surgical team. In addition, dynamic CT scan has been reported to have higher sensitivity than transabdominal US and is not limited by the tumor's location in the body or tail of the pancreas. If CT scan fails to localize the tumor or if there are concurrent hepatic lesions that cannot be adequately visualized on CT, we proceed with an MRI as an adjuvant modality. As a second step, we universally employ EUS to better evaluate the tumor's location in relation to relevant anatomic structures such as the pancreatic duct and major blood vessels. EUS in addition allows for detection of lesions that may not be apparent on dynamic CT scan, especially at the head of the pancreas. Preoperative tattooing is always employed regardless of the availability of IOUS or a planned laparoscopic or open procedure. In our experience, in the overwhelming majority of cases these preoperative imaging modalities are sufficient for accurate localization and subsequent surgical planning.

In most cases, the combination of various invasive and non-invasive modalities suffices to accurately determine preoperatively the anatomical location of sporadic insulinomas. In MEN1 special attention is focused on evaluating the presence or not of insulinomas or other PanNETs in the head of the pancreas, since in most cases distal pancreatectomy will be the procedure followed, thereby obviating the need for precise localization of lesions in the body or tail of the pancreas.

However, in the era of laparoscopic surgery, preoperative planning has limitations during the actual surgical procedure. During the years before minimally invasive techniques, a relatively high percentage of insulinomas (as much as 10\%) remained unidentifiable by bimanual palpation of the pancreas alone. ${ }^{44,45}$ Intraoperative ultrasound (IOUS) is an invaluable and irreplaceable tool when pursuing both open and laparoscopic enucleation. ${ }^{15,46}$ In open exploration, the combination of preoperative localization and intraoperative palpation and IOUS can achieve successful surgical resection in virtually all patients $(83 \%$ to $98 \%) .{ }^{15,47}$ In a similar manner to EUS, it can relay information regarding tumor location and proximity to the pancreatic duct. ${ }^{48}$ In several series its sensitivity in localizing insulinoma is higher than $90 \%$ and considered superior to preoperative imaging studies, it being able to detect lesions as small as $2-3 \mathrm{~mm} .{ }^{15,49-51}$ It is hence is easy to understand that in laparoscopic surgery where there is typically a loss of tactile sensation, IOUS gains even greater importance with results being comparable to IOUS in open surgery. ${ }^{52-54} \mathrm{~A}$ lack of laparoscopic IOUS, despite positive preoperative identification, has been cited as an important factor in the failure to intraoperatively identify lesions leading to increased incidences of conversion to open procedure. ${ }^{55,56}$ It should be noted, however, that even though IOUS boasts higher identification rates than the preoperative setting, there have been reports where laparoscopic IOUS has failed to detect a tumor which was positively identified in preoperative imaging. ${ }^{57}$

\section{DETERMINATION OF OPERATIVE TECHNIQUE}

Surgical excision of insulinoma remains the treatment of choice. ${ }^{4,13,14}$ Traditional resections and parenchyma-sparing resections or a combination of both can be employed depending on indications. Factors that dictate the choice of procedure and should be addressed preoperatively are a diagnosis or not of 
MEN1, the number of insulinomas, the tumor's size, location in the pancreas and anatomical proximity to the major pancreatic duct and major blood vessels, and the likelihood of malignancy. As previously stated, the majority of insulinomas are sporadic and in turn most sporadic insulinomas are solitary and benign. Therefore, parenchyma-sparing procedures such as enucleation and central pancreatectomy are frequently performed in the management of sporadic insulinoma. Conversely, in MEN1 patients, due to the fact that the disease is regularly multifocal, a different strategy is employed and a distal pancreatectomy is the standard of care with or without enucleation of masses in the head of the pancreas.

\section{OPEN OR LAPAROSCOPIC APPROACH?}

Over the last two decades, minimally invasive techniques have been employed in the treatment of PanNETs with various levels of success..$^{51,58-61}$ Insulinomas constitute a distinct category, since the majority are benign, solitary and usually small in size at the time of diagnosis $(<2 \mathrm{~cm})$. Thus, common sense dictates that they are more amenable to laparoscopic intervention than other PanNETs. Despite the fact that insulinomas are the most common functional PanNET, they still remain a rare clinical entity and consequently even large volume centers have a limited number of cases that are managed laparoscopically. ${ }^{51,57,60,62}$ To our knowledge, no randomized control studies comparing open and laparoscopic management of insulinomas have been performed and are probably unlikely to be performed due to the paucity of cases. The majority of reports that attempt to make direct comparisons are retrospective in nature and therefore have certain limitations..$^{56,57,59,63}$ Due to the relatively small number of cases there is difficulty in demonstrating significant differences in important issues such as complications rates. ${ }^{19}$ Multicentric studies are plagued by interinstitution variability in preoperative management and expertise in advanced laparoscopic techniques. ${ }^{64}$ In addition, heterogeneity in most of these studies renders comparisons between open and laparoscopic approaches problematic. ${ }^{65}$ In certain large-scale studies, additional pancreatic pathologies are included thus making generalization of results difficult. ${ }^{59,66-68}$ Furthermore, since there are no randomized studies, these reports are susceptible to selection bias in regard to the patients who were chosen to undergo laparoscopic intervention. Of note is also the fact that laparoscopic management of PanNETs is still a nascent discipline and most published studies have been undertaken in referral institutions with considerable experience in neuroendocrine pancreatic surgery and advanced laparoscopic techniques and that have access to various preoperative and intraoperative diagnostic modalities. Hence, it remains unclear if their findings can be extrapolated to other institutions which have extensive experience in open pancreatic surgery and other advanced laparoscopic procedures.

On a practical level, pancreatic surgery is technically vexing for a variety of reasons. The pancreas has a retroperitoneal position, in close proximity to major vessels and with a very fragile consistency that renders it extremely vulnerable to any manipulation (Figure 3). Laparoscopy adds another layer of complexity eliminating tactile sensation and thus rendering identification of anatomical structures harder. Obviously, these types of procedures require extensive familiarity with open pancreatic surgery combined with a high level of laparoscopic dexterity. It is noteworthy that both open and laparoscopic resection of insulinoma share similar operative strategy.

What can be deduced from previous studies is that laparoscopic surgical management of insulinomas is feasible and safe in selected patients in institutions that have a high volume of PanNET cases. Randomized control studies are difficult to implement due to the rarity of the disease.

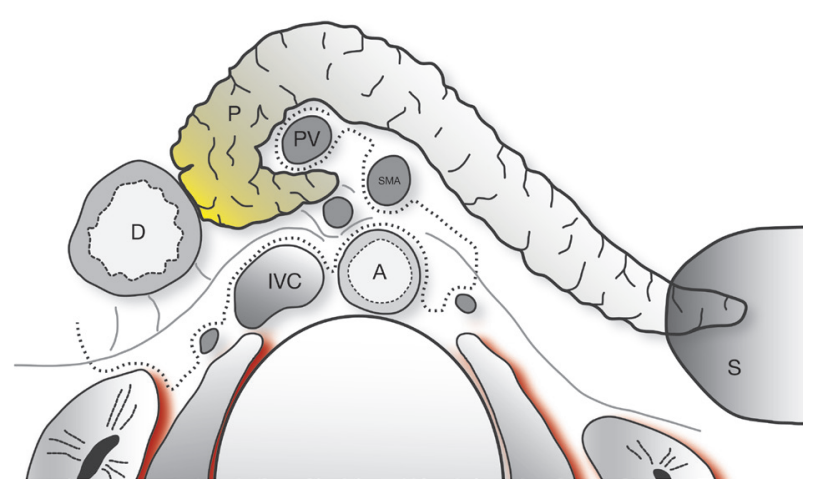

Figure 3. Transverse section of the pancreas indicating anatomic relations with vital anatomic structures. (P: Pancreas; D: Duodenum; PV: Portal Vein; IVC: Inferior Vena Cava; A: Aorta; SMA: Superior Mesenteric Artery; S: Spleen). 


\section{SURGICAL OPTIONS IN LAPAROSCOPY}

The first laparoscopic attempts at insulinoma excision performed 20 years ago indicated that laparoscopic management was feasible and with an acceptable safety profile. ${ }^{61}$ Since then, numerous highly specialized centers have accrued experience in advanced laparoscopic techniques in pancreatic surgery and in these places most if not all procedures can be completed laparoscopically. ${ }^{51,57,62,69}$ Pancreaticoduodenectomy remains a relative exception due to its complexity but is technically feasible in specialized institutions presenting acceptable results compared to open pancreaticoduodenectomy. ${ }^{70,71}$ As a result, in high volume centers laparoscopic management has become the mainstay of surgical treatment due to several factors, including shorter length of stay and better cosmetic results. Most cases in the literature involve enucleation of solitary lesions in the body or tail of the pancreas and distal pancreatectomy with or without spleen preservation. ${ }^{51,59,62}$ A recent meta-analysis of large-scale studies indicated that approximately $65 \%$ of laparoscopic procedures completed were enucleations and about $34 \%$ distal pancreatectomies, the remainder being pancreaticoduodenectomy and central pancreatectomy. ${ }^{19}$ Therefore, here we will focus on enucleation and distal pancreatectomy with or without spleen preservation in patients with sporadic insulinomas. MEN1-associated insulinomas represent a different type of surgical challenge for reasons that were alluded to previously. MEN1-associated insulinomas have a high malignancy potential and are frequently multifocal, while to a large extent there are other PanNETs concurrently present. However, several series have indicated that minimally invasive procedures can be employed with satisfactory results compared to open procedures..$^{51,69,72}$

Current treatment guidelines strongly recommend enucleation in sporadic solitary insulinoma with a diameter $<2 \mathrm{~cm}$ on IOUS and if structural integrity of the pancreatic duct can be maintained. ${ }^{14}$ A $2-3 \mathrm{~mm}$ distance from the pancreatic duct verified on IOUS is currently advised when attempting enucleation. ${ }^{13,73,74}$ Larger tumors up to $3 \mathrm{~cm}$ carry an increased risk for malignancy and increased rates of postoperative complications have been reported, thus consensus is lacking as to which approach is optimal..$^{57,59,75}$ Past series have reported successful application of lapa- roscopic techniques in the management of sporadic insulinomas located especially in the body or tail of the pancreas. ${ }^{51,60,62,69}$ Concerns giving rise to debate still persist in the management of solitary lesions located in the head of the pancreas due to complications (up to $64 \%$ may develop a fistula) after enucleation. ${ }^{76,77}$ A review of the literature indicates that laparoscopic enucleation when employed in selected patients is a technically feasible procedure with comparable complication rates..$^{51,57,63,71}$ The most common surgical complication is pancreatic fistula $(7.2 \%$ in all types of laparoscopic procedures) with the majority occurring in laparoscopic enucleations. ${ }^{19}$ Rates of pancreatic fistula after laparoscopic enucleation for various pathologies are variable (13\%-56\%) and may depend on the location of the lesion and its pathology ${ }^{66,68,77}$ In early reports, proximity to major vessels such as the portal vein was considered a relative contraindication to laparoscopic enucleation. ${ }^{19}$ Recent case reports indicate that laparoscopic enucleation is feasible even in perilous anatomic positions, such as the posterior surface of the neck of the pancreas, indicating that with increased familiarity the extent of contraindications for laparoscopic enucleation is shrinking. ${ }^{78}$

Traditional pancreatectomies are usually carried out for solitary insulinomas when adequate distance from the pancreatic duct is not present. Distal or subtotal pancreatectomies are also performed in MEN1 procedures due to frequent multifocal disease, concurrent presence of other PanNETs and potential for underlying malignancy.

Laparoscopic spleen-preserving pancreatectomy is gaining traction as a safe procedure. ${ }^{69,79,80}$ There are two approaches to spleen-preserving pancreatectomy, one entailing splenic vessel preservation and an alternative technique with splenic vessel resection. ${ }^{81,82}$ Due to the usually benign nature of insulinomas, splenic vessel preservation is advocated as a first-line treatment option. ${ }^{80,83}$ The spleen's viability in splenic vessel resection is dependent on the blood supply of the short gastric vessels. Rates of splenic infarction are more common in this type of procedure but whether there is any clinical relevance remains to be seen. ${ }^{83-85}$

Here we briefly outline certain technical considerations which are of importance when undertaking 
laparoscopic management of insulinomas during enucleation and distal pancreatectomy. Due to the low frequency of laparoscopic pancreaticoduodenectomy and central pancreatectomy, these will not be elaborated upon.

\section{TECHNICAL ASPECTS}

\section{Insulinomas of the body or tail of the pancreas}

Insulinomas tend to be compact and encapsulated, presenting a clear dissection plane between the tumor and the surrounding pancreas. ${ }^{27}$ It is important to remove the tumor with the capsule completely to prevent a local recurrence. Once the tumor has been located and the relationship to the pancreatic duct has been defined, the decision for laparoscopic enucleation or distal pancreatectomy should be made. Tumors in the anterior or inferior surface of the pancreas require minimal mobilization, while lesions in the posterior or superior surface demand adequate mobilization of the body and tail. So if the lesion is visible on the anterior surface of the pancreas and appropriate for enucleation, it is dissected from the surrounding pancreatic tissue with diathermy or laparoscopic vessel sealing device (Figure 4). If the insulinoma is buried within the pancreas, the pancreatic tissue is opened at the appropriate site, just above the lesion, and the insulinoma is enucleated carefully, without causing any damage to the pancreatic duct. For lesions in the posterior surface, lifting of the inferior margin of the body of the pancreas is performed and if the splenic vein is in close proximity care is taken to isolate it from the tumor. Occasionally, enucleation is only possible after local resection of the adjacent portion of the vein. In this process, injury to the splenic artery must be avoided. After enucleation, the tumor bed must be examined for evidence of pancreatic duct injury. Tumors situated very distally in the splenic hilum are especially difficult to identify. In cases where visualization and ultrasound fail, a hand port can be used to allow palpation of the gland.

Insulinomas located in the distal portion of the tail of the pancreas or in very close proximity to the main pancreatic duct $(<3 \mathrm{~mm})$ require distal pancreatectomy. ${ }^{13}$ The technique of laparoscopic distal pancreatectomy varies widely between different centers

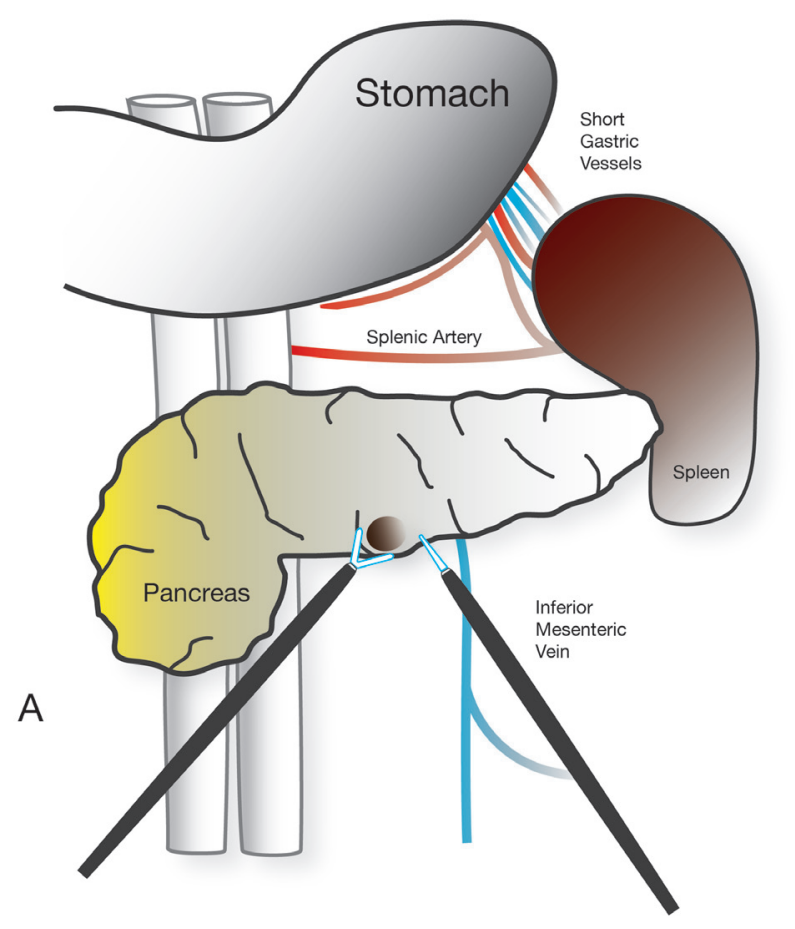

Figure 4. Enucleation of an insulinoma on the inferior border of the body of the pancreas.

and surgeons. Distal pancreatectomy can be combined with splenectomy and with spleen preservation with or without preservation of the splenic vessels (Figure 5 and 6). ${ }^{81}$ The key vascular structures in this operation are the superior mesenteric vein, splenic vein and artery, hepatic artery and inferior mesenteric vein. The anatomic landmark for medial mobilization of the pancreas is the superior mesenteric vein (SMV). Small and distal lesions do not require formal left pancreatectomy and in these cases the pancreas may be divided medial to the SMV. Once the pancreas is free inferiorly from its peritoneal attachments, from the SMV to the splenic hilum, dissection under the pancreas may be performed. In distal pancreatectomy with splenectomy the splenic vessels are divided. The splenic artery is stapled at any point during the operation. Ideally it is ligated a few centimeters beyond its origin at the celiac axis. It is important not to injure the hepatic artery during the ligation of the splenic artery. The splenic vessels can be divided along with the pancreas or separately depending on the thickness of the pancreatic parenchyma using lineal staplers.

Spleen preserving distal pancreatectomy is cur- 


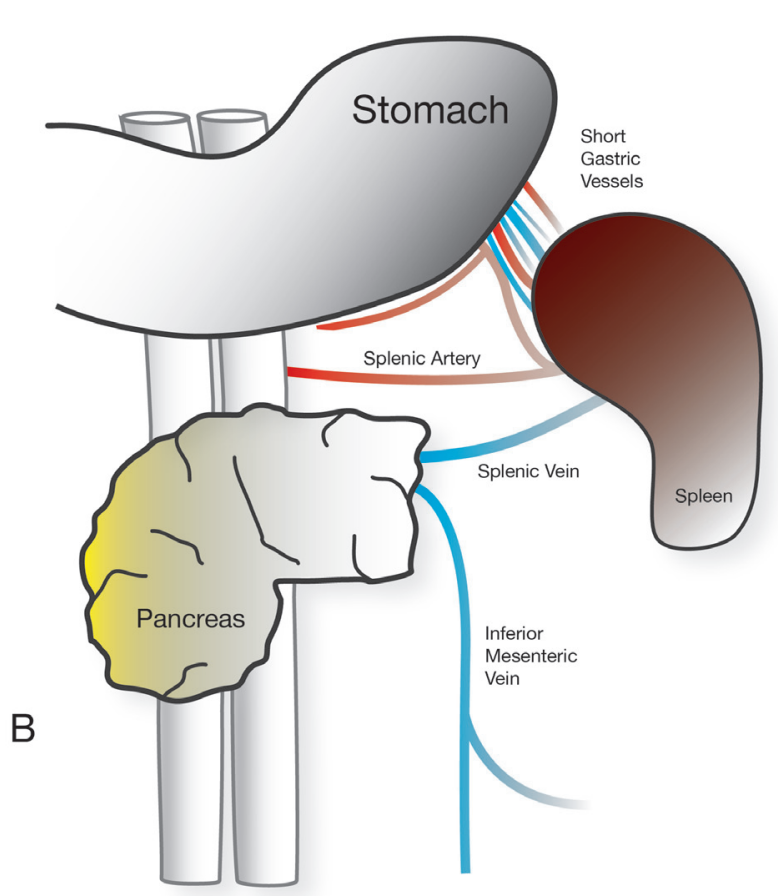

Figure 5. Spleen preserving distal pancreatectomy with preservation of the splenic vessels.

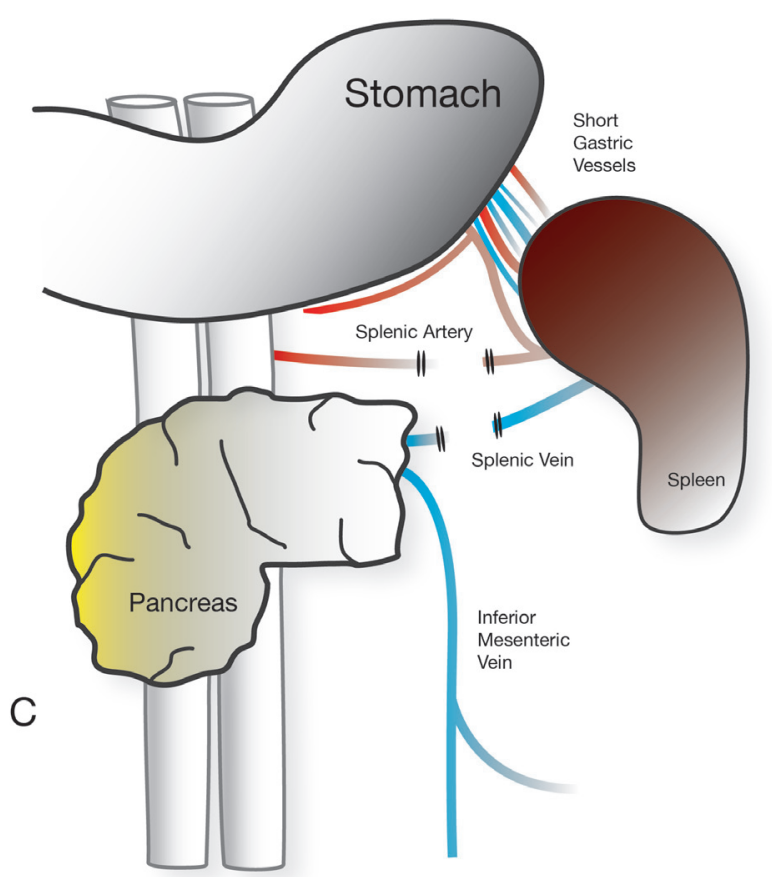

Figure 6. Spleen preserving distal pancreatectomy with resection of splenic vessels.

rently the gold standard in benign pancreatic conditions, even though they are technically demanding.
In 1996, Kimura et al described a technique of spleen preserving distal pancreatectomy with preservation of the splenic artery and vein. ${ }^{82}$ Earlier, in 1988, Warshaw had described a technique of spleen-preserving distal pancreatectomy with transection of the splenic vessels. ${ }^{81}$ During these operations care should be taken not to ligate the short gastric and the left gastrepiploic vessels. After dissecting the inferior pancreatic border from the retroperitoneum, visualization of the posterior surface of the pancreas is feasible. The SMV is identified as is the splenic vein since they form the portal vein. The splenic vessels have to be dissected circumferentially and have to be separated carefully from the pancreas. With the Warshaw technique, the splenic vessels are ligated at the side of the portomesenteric vein and the splenic hilum. After this procedure the sole remaining blood supply to the spleen is from the gastric and the left gastroepiploic vessels. The left gastroepiploic artery is suspected to play an important role in the prevention of postoperative ischemia of the spleen.

It is obvious that during a spleen-preserving operation the surgeon should always examine the splenic perfusion at the end of the procedure. A splenectomy has to be performed when signs of splenic ischemia are present.

\section{INSULINOMAS OF THE HEAD OF THE PANCREAS}

The excisions of insulinomas of the pancreatic head are technically more demanding. An extensive Kocher maneuver is carried out to mobilize the head of the pancreas in insulinomas on both the anterior surface and posterior surface of the head. After appropriate exposure of the pancreas IOUS is performed. IOUS is used to identify the location of the tumor and its relation to the main pancreatic duct and the SMV. For tumors of the pancreatic head a hand port may be placed in the right subcostal area to help with mobilization and gain the tactile sensation. Enucleations performed on the posterior surface of the head require extensive mobilization of the right hepatic lobe and right kidney. Lesions on the anterior surface of the head require extensive identification of various blood vessels. For lesions located along the inferior border of the head, the head is separated from the SMV and 
portal vein by ligating the small collaterals vessels. For tumors along the superior edge of the pancreatic head, the common hepatic artery is separated from the upper border of the pancreas. Enucleation is performed from the pancreatic parenchyma using again diathermy or laparoscopic vessel sealing device. In all of these operations, after the enucleation the tumor bed is carefully examined and a drain is left in place.

\section{CONCLUSIONS}

Insulinomas are rare tumors of the pancreas which mostly occur sporadically. They are commonly of a benign nature and respond excellently to surgical excision. From a surgical standpoint, localization of the tumor is of critical importance. Successful identification eliminates the need for blind resections of pancreatic tissue. However, no consensus has been attained for the preoperative localization of these tumors. Local availability and skill in specific invasive and non-invasive tests seem to determine the preoperative algorithm. Advances in minimally invasive surgical techniques have for the last two decades permitted the introduction of laparoscopic surgery in the treatment of this condition. Results have been encouraging and comparable to those of open surgical techniques. However, there is an absence of randomized control studies that would conclusively verify the conclusions of these studies. Another question that has not been addressed is whether these results can be reproducible in institutions that are adept in open pancreatic surgery and other advanced laparoscopic procedures but do not encounter a large number of insulinomas in their surgical workload. From a technical standpoint, the same basic principles that dictate open surgical management are applicable to laparoscopic treatment. Identification and visualization of several vital structures is mandatory as is excellent knowledge of the anatomical landmarks by the surgical team. In its current state, laparoscopic management of insulinomas is a safe and reasonable option in high volume centers specializing in laparoscopic pancreatic surgery. Availability of several preoperative imaging modalities and intraoperative US is mandatory when considering the laparoscopic approach to insulinomas. As has been the case with other advanced laparoscopic procedures, diffusion of knowledge and experience will doubtless make these types of procedures more common in the near future.

\section{CONFLICT OF INTEREST STATEMENT}

The authors have no conflict of interest to declare.

\section{REFERENCES}

1. Metz DC, Jensen RT, 2008 Gastrointestinal neuroendocrine tumors: pancreatic endocrine tumors. Gastroenterology 135: 1469-1492.

2. Halfdanarson TR, Rubin J, Farnell MB, Grant CS, Petersen GM, 2008 Pancreatic endocrine neoplasms: epidemiology and prognosis of pancreatic endocrine tumors. Endocr Relat Cancer 15: 409-427.

3. Lam KY, Lo CY, 1997 Pancreatic endocrine tumour: a 22-year clinico-pathological experience with morphological, immunohistochemical observation and a review of the literature. Eur J Surg Oncol 23: 36-42.

4. Service FJ, McMahon MM, O’Brien PC, Ballard DJ, 1991 Functioning insulinoma-Incidence, recurrence, and long-term survival of patients: a 60-year study. Mayo Clin Proc 66: 711-719.

5. Jensen RT, Berna MJ, Bingham DB, Norton JA, 2008 Inherited pancreatic endocrine tumor syndromes: advances in molecular pathogenesis, diagnosis, management, and controversies. Cancer 113: 1807-1843.

6. Demeure MJ, Klonoff DC, Karam JH, Duh QY, Clark OH, 1991 Insulinomas associated with multiple endocrine neoplasia type I: the need for a different surgical approach. Surgery 110: 998-1004.

7. Vaidakis D, Karoubalis J, Pappa T, Piaditis G, Zografos GN, 2010 Pancreatic insulinoma: current issues and trends. Hepatobiliary Pancreat Dis Int 9: 234-241.

8. Bartsch DK, Albers M, Knoop R, Kann PH, Fendrich V, Waldmann J, 2013 Enucleation and limited pancreatic resection provide long-term cure for insulinoma in multiple endocrine neoplasia type 1 . Neuroendocrinology 98: 290-298.

9. de Herder WW, Niederle B, Scoazec JY, et al, 2006 Well-differentiated pancreatic tumor/carcinoma: insulinoma. Neuroendocrinology 84: 183-188.

10. Placzkowski KA, Vella A, Thompson GB, et al, 2009 Secular trends in the presentation and management of functioning insulinoma at the Mayo Clinic, 1987-2007. J Clin Endocrinol Metab 94: 1069-1073.

11. Boukhman MP, Karam JH, Shaver J, Siperstein AE, Duh QY, Clark OH, 1998 Insulinoma-experience from 1950 to 1995 . West J Med 169: 98-104.

12. Mathur A, Gorden P, Libutti SK, 2009 Insulinoma. Surg Clin North Am 89: 1105-1121.

13. Jensen RT, Cadiot G, Brandi ML, et al, 2012 ENETS Consensus Guidelines for the management of patients with digestive neuroendocrine neoplasms: functional pancreatic endocrine tumor syndromes. Neuroendo- 
crinology 95: 98-119.

14. Kulke MH, Anthony LB, Bushnell DL, et al, 2010 NANETS treatment guidelines: well-differentiated neuroendocrine tumors of the stomach and pancreas. Pancreas 39: 735-752.

15. Goh BK, Ooi LL, Cheow PC, et al, 2009 Accurate preoperative localization of insulinomas avoids the need for blind resection and reoperation: analysis of a single institution experience with 17 surgically treated tumors over 19 years. J Gastrointest Surg 13: 10711077.

16. Kuzin NM, Egorov AV, Kondrashin SA, Lotov AN, Kuznetzov NS, Majorova JB, 1998 Preoperative and intraoperative topographic diagnosis of insulinomas. World J Surg 22: 593-597; discussion 7-8.

17. Lo CY, Tam PK, 2003 Laparoscopic pancreatic resection of an insulinoma in a child. Asian J Surg 26: 43-45.

18. An L, Li W, Yao KC, et al, 2011 Assessment of contrastenhanced ultrasonography in diagnosis and preoperative localization of insulinoma. Eur J Radiol 80: 675-680.

19. Mehrabi A, Fischer L, Hafezi M, et al, 2014 A systematic review of localization, surgical treatment options, and outcome of insulinoma. Pancreas 43: 675-686.

20. Gouya H, Vignaux O, Augui J, et al, 2003 CT, endoscopic sonography, and a combined protocol for preoperative evaluation of pancreatic insulinomas. AJR Am J Roentgenol 181: 987-992.

21. Nikfarjam M, Warshaw AL, Axelrod L, et al, 2008 Improved contemporary surgical management of insulinomas: a 25-year experience at the Massachusetts General Hospital. Ann Surg 247: 165-172.

22. Zhang T, Mu Y, Qu L, et al, 2012 Accurate combined preoperative localization of insulinomas aid the choice for enucleation: a single institution experience over 25 years. Hepatogastroenterology 59: 1282-1285.

23. Tamm EP, Bhosale P, Lee JH, Rohren EM, 2016 Stateof-the-art imaging of pancreatic neuroendocrine tumors. Surg Oncol Clin N Am 25: 375-400.

24. Druce MR, Muthuppalaniappan VM, O’Leary B, et al, 2010 Diagnosis and localisation of insulinoma: the value of modern magnetic resonance imaging in conjunction with calcium stimulation catheterisation. Eur J Endocrinol 162: 971-978.

25. Thoeni RF, Mueller-Lisse UG, Chan R, Do NK, Shyn PB, 2000 Detection of small, functional islet cell tumors in the pancreas: selection of MR imaging sequences for optimal sensitivity. Radiology 214: 483-490.

26. Mirallie E, Pattou F, Malvaux P, et al, 2002 Value of endoscopic ultrasonography and somatostatin receptor scintigraphy in the preoperative localization of insulinomas and gastrinomas. Experience of 54 cases. Gastroenterol Clin Biol 26: 360-366.

27. Grant CS, 2005 Insulinoma. Best Pract Res Clin Gastroenterol 19: 783-798.

28. Brown CK, Bartlett DL, Doppman JL, et al, 1997 Intraarterial calcium stimulation and intraoperative ultrasonography in the localization and resection of insulinomas. Surgery 122: 1189-1193; discussion 9394.

29. Winter TC, 3rd, Nghiem HV, Freeny PC, Hommeyer SC, Mack LA, 1995 Hepatic arterial anatomy: demonstration of normal supply and vascular variants with three-dimensional CT angiography. Radiographics 15: 771-780.

30. Dravecka I, Lazurova I, 2014 Nesidioblastosis in adults. Neoplasma 61: 252-256.

31. Thompson SM, Vella A, Thompson GB, et al, 2015 Selective Arterial Calcium Stimulation With Hepatic Venous Sampling Differentiates Insulinoma From Nesidioblastosis. J Clin Endocrinol Metab 100: 4189-4197.

32. Guettier JM, Kam A, Chang R, et al, 2009 Localization of insulinomas to regions of the pancreas by intraarterial calcium stimulation: the NIH experience. J Clin Endocrinol Metab 94: 1074-1080.

33. Anderson MA, Carpenter S, Thompson NW, Nostrant TT, Elta GH, Scheiman JM, 2000 Endoscopic ultrasound is highly accurate and directs management in patients with neuroendocrine tumors of the pancreas. Am J Gastroenterol 95: 2271-2277.

34. Tellez-Avila FI, Acosta-Villavicencio GY, Chan C, et al, 2015 Diagnostic yield of endoscopic ultrasound in patients with hypoglicemia and insulinoma suspected. Endosc Ultrasound 4: 52-55.

35. Puli SR, Kalva N, Bechtold ML, et al, 2013 Diagnostic accuracy of endoscopic ultrasound in pancreatic neuroendocrine tumors: a systematic review and meta analysis. World J Gastroenterol 19: 3678-3684.

36. Kann PH, 2016 Endoscopic Ultrasound in Endocrinology: Imaging of the Adrenals and the Endocrine Pancreas. Front Horm Res 45: 46-54.

37. Sotoudehmanesh R, Hedayat A, Shirazian N, et al, 2007 Endoscopic ultrasonography (EUS) in the localization of insulinoma. Endocrine 31: 238-241.

38. Joseph AJ, Kapoor N, Simon EG, et al, 2013 Endoscopic ultrasonography--a sensitive tool in the preoperative localization of insulinoma. Endocr Pract 19: 602-608.

39. Leelasinjaroen P, Manatsathit W, Berri R, Barawi M, Gress FG, 2014 Role of preoperative endoscopic ultrasound-guided fine-needle tattooing of a pancreatic head insulinoma. World J Gastrointest Endosc 6: 506-509.

40. Newman NA, Lennon AM, Edil BH, et al, 2010 Preoperative endoscopic tattooing of pancreatic body and tail lesions decreases operative time for laparoscopic distal pancreatectomy. Surgery 148: 371-377.

41. Zografos GN, Stathopoulou A, Mitropapas G, et al, 2005 Preoperative imaging and localization of small sized insulinoma with EUS-guided fine needle tattoing: a case report. Hormones (Athens) 4: 111-116.

42. Zografos GN, Vasiliadis G, Karoubalis J, Tsagarakis S, 2010 Laparoscopic resection of insulinoma after endoscopic ultrasonography tattooing. Am Surg 76: 446-448. 
43. Levy MJ, Thompson GB, Topazian MD, Callstrom MR, Grant CS, Vella A, 2012 US-guided ethanol ablation of insulinomas: a new treatment option. Gastrointest Endosc 75: 200-206.

44. Kaplan EL, Lee CH, 1979 Recent advances in the diagnosis and treatment of insulinomas. Surg Clin North Am 59: 119-129.

45. Hirshberg B, Libutti SK, Alexander HR, et al, 2002 Blind distal pancreatectomy for occult insulinoma, an inadvisable procedure. J Am Coll Surg 194: 761-764.

46. Grover AC, Skarulis M, Alexander HR, et al, 2005 A prospective evaluation of laparoscopic exploration with intraoperative ultrasound as a technique for localizing sporadic insulinomas. Surgery 138: 1003-1008.

47. Norton JA, Cromack DT, Shawker TH, et al, 1988 Intraoperative ultrasonographic localization of islet cell tumors. A prospective comparison to palpation. Ann Surg 207: 160-168.

48. Shin LK, Brant-Zawadzki G, Kamaya A, Jeffrey RB, 2009 Intraoperative ultrasound of the pancreas. Ultrasound Q 25: 39-48.

49. Galiber AK, Reading CC, Charboneau JW, et al, 1988 Localization of pancreatic insulinoma: comparison of pre- and intraoperative US with CT and angiography. Radiology 166: 405-408.

50. Hiramoto JS, Feldstein VA, LaBerge JM, Norton JA, 2001 Intraoperative ultrasound and preoperative localization detects all occult insulinomas; discussion 1025-6. Arch Surg 136: 1020-1025.

51. Fernandez-Cruz L, Blanco L, Cosa R, Rendon H, 2008 Is laparoscopic resection adequate in patients with neuroendocrine pancreatic tumors? World J Surg 32: 904-917.

52. Berends FJ, Cuesta MA, Kazemier G, et al, 2000 Laparoscopic detection and resection of insulinomas. Surgery 128: 386-391.

53. Iihara M, Kanbe M, Okamoto T, Ito Y, Obara T, 2001 Laparoscopic ultrasonography for resection of insulinomas. Surgery 130: 1086-1091.

54. Jaroszewski DE, Schlinkert RT, Thompson GB, Schlinkert DK, 2004 Laparoscopic localization and resection of insulinomas. Arch Surg 139: 270-274.

55. Ayav A, Bresler L, Brunaud L, Boissel P, 2005 Laparoscopic approach for solitary insulinoma: a multicentre study. Langenbecks Arch Surg 390: 134-140.

56. Sa Cunha A, Beau C, Rault A, Catargi B, Collet D, Masson B, 2007 Laparoscopic versus open approach for solitary insulinoma. Surg Endosc 21: 103-108.

57. Hu M, Zhao G, Luo Y, Liu R, 2011 Laparoscopic versus open treatment for benign pancreatic insulinomas: an analysis of 89 cases. Surg Endosc 25: 3831-3837.

58. Cauley CE, Pitt HA, Ziegler KM, et al, 2012 Pancreatic enucleation: improved outcomes compared to resection. J Gastrointest Surg 16: 1347-1353.

59. DiNorcia J, Lee MK, Reavey PL, et al, 2010 One hundred thirty resections for pancreatic neuroendocrine tumor: evaluating the impact of minimally invasive and parenchyma-sparing techniques. J Gastrointest Surg 14: 1536-1546.

60. Fernandez-Cruz L, Herrera M, Saenz A, Pantoja JP, Astudillo E, Sierra M, 2001 Laparoscopic pancreatic surgery in patients with neuroendocrine tumours: indications and limits. Best Pract Res Clin Endocrinol Metab 15: 161-175.

61. Gagner M, Pomp A, Herrera MF, 1996 Early experience with laparoscopic resections of islet cell tumors. Surgery 120: 1051-1054.

62. Isla A, Arbuckle JD, Kekis PB, et al, 2009 Laparoscopic management of insulinomas. Br J Surg 96: 185-190.

63. Zhao YP, Zhan HX, Zhang TP, et al, 2011 Surgical management of patients with insulinomas: Result of 292 cases in a single institution. J Surg Oncol 103: 169-174.

64. Zerbi A, Capitanio V, Boninsegna L, et al, 2011 Surgical treatment of pancreatic endocrine tumours in Italy: results of a prospective multicentre study of 262 cases. Langenbecks Arch Surg 396: 313-321.

65. Drymousis P, Raptis DA, Spalding D, et al, 2014 Laparoscopic versus open pancreas resection for pancreatic neuroendocrine tumours: a systematic review and metaanalysis. HPB (Oxford) 16: 397-406.

66. Dedieu A, Rault A, Collet D, Masson B, Sa Cunha A, 2011 Laparoscopic enucleation of pancreatic neoplasm. Surg Endosc 25: 572-576.

67. Rosok BI, Marangos IP, Kazaryan AM, et al, 2010 Single-centre experience of laparoscopic pancreatic surgery. Br J Surg 97: 902-909.

68. Fernandez-Cruz L, Cosa R, Blanco L, Levi S, LopezBoado MA, Navarro S, 2007 Curative laparoscopic resection for pancreatic neoplasms: a critical analysis from a single institution. J Gastrointest Surg 11: 16071621; discussion 21-22.

69. Fernandez-Cruz L, Martinez I, Cesar-Borges G, et al, 2005 Laparoscopic surgery in patients with sporadic and multiple insulinomas associated with multiple endocrine neoplasia type 1. J Gastrointest Surg 9: 381-388.

70. Correa-Gallego C, Dinkelspiel HE, Sulimanoff I, et al, 2014 Minimally-invasive vs open pancreaticoduodenectomy: systematic review and meta-analysis. J Am Coll Surg 218: 129-139.

71. Mabrut JY, Fernandez-Cruz L, Azagra JS, et al, 2005 Laparoscopic pancreatic resection: results of a multicenter European study of 127 patients. Surgery 137: 597-605.

72. Lopez CL, Albers MB, Bollmann C, et al, 2016 Minimally invasive versus open pancreatic surgery in patients with multiple endocrine neoplasia type 1. World J Surg [Epub ahead of print].

73. Brient C, Regenet N, Sulpice L, et al, 2012 Risk factors for postoperative pancreatic fistulization subsequent to enucleation. J Gastrointest Surg 16: 1883-1887. 
74. Heeger K, Falconi M, Partelli S, et al, 2014 Increased rate of clinically relevant pancreatic fistula after deep enucleation of small pancreatic tumors. Langenbecks Arch Surg 399: 315-321.

75. Pitt SC, Pitt HA, Baker MS, et al, 2009 Small pancreatic and periampullary neuroendocrine tumors: resect or enucleate? J Gastrointest Surg 13: 1692-1698.

76. Jilesen AP, van Eijck CH, Busch OR, van Gulik TM, Gouma DJ, Nieveen van Dijkum EJ, 2015 Postoperative Outcomes of Enucleation and Standard Resections in Patients with a Pancreatic Neuroendocrine Tumor. World J Surg 40: 715-728.

77. Costi R, Randone B, Mal F, Basato S, Levard H, Gayet B, 2013 A critical appraisal of laparoscopic pancreatic enucleations: right-sided procedures (Pancreatic Head, Uncus) are not mini-invasive surgery. Surg Laparosc Endosc Percutan Tech 23: 524-531.

78. Conrad C, Passot G, Katz MH, et al, 2016 Laparoscopic insulinoma enucleation from the retro-pancreatic neck: a stepwise approach. Ann Surg Oncol 23: 2001.

79. Sciuto A, Abete R, Reggio S, Pirozzi F, Settembre A, Corcione F, 2014 Laparoscopic spleen-preserving distal pancreatectomy for insulinoma: experience of a single center. Int J Surg 12: Suppl 1: 152-155.
80. Lv GY, Wang GY, Jiang C, et al, 2014 Laparoscopic spleen-preserving distal pancreatectomy with or without splenic vessel conservation: a retrospective study of 20 cases. Hepatogastroenterology 60: 1785-1788.

81. Warshaw AL, 1988 Conservation of the spleen with distal pancreatectomy. Arch Surg 123: 550-553.

82. Kimura W, Inoue T, Futakawa N, Shinkai H, Han I, Muto T, 1996 Spleen-preserving distal pancreatectomy with conservation of the splenic artery and vein. Surgery 120: 885-890.

83. Zhou ZQ, Kim SC, Song KB, Park KM, Lee JH, Lee YJ, 2014 Laparoscopic spleen-preserving distal pancreatectomy: comparative study of spleen preservation with splenic vessel resection and splenic vessel preservation. World J Surg 38: 2973-2979.

84. Kim H, Song KB, Hwang DW, et al, 2016 A single-center experience with the laparoscopic Warshaw technique in 122 consecutive patients. Surg Endosc [Epub ahead of print].

85. Partelli S, Cirocchi R, Randolph J, Parisi A, Coratti A, Falconi M, 2016 A systematic review and metaanalysis of spleen-preserving distal pancreatectomy with preservation or ligation of the splenic artery and vein. Surgeon 14: 109-118. 